\title{
Ventrículo derecho: Relación con patología respiratoria y patología del ventrículo izquierdo
}

\author{
IVÁN CAVIEDES S.*, POLENTZI URIARTE G. DE C.** y CRISTIÁN LÓPEZ H.***
}

\section{Right ventricle: Relationship with pulmonary and left ventricular pathology}

In a previous article we reviewed pathophysiological concepts of right ventricle considering its importance in cardiorespiratory diseases and its behavior was compared with left ventricle. Measurement of right ventricular function is complex due to its pre load dependence. Remarkable progress has been developed in methods to measure anatomy and function. Probably a functional definition might include diverse parameters as geometry, hypertrophy, dilatation, contraction, oxygen supply to muscular wall and ejected volume. In the present review we describe the behavior of right ventricle in different pathologies. In first place we analyze its behavior in respiratory diseases and afterwards in diseases which involves pulmonary circulation and left ventricular failure. Our purpose is to stimulate pulmonary physicians and cardiologists in the study of this common entity. A complete description of the diseases which causes right ventricular failure goes beyond the limits of this article.

Key words: Right ventricle, right ventricle failure, cor pulmonale.

\section{Resumen}

En una publicación previal actualizamos los conceptos fisiopatológicos, que rigen la función del ventrículo derecho (VD), considerando su importancia en enfermedades cardiorespiratorias, y describimos su comportamiento al compararlo con el ventrículo izquierdo (VI). La función del VD es dificil de medir por su alta dependencia de la precarga, sin embargo, ha habido progresos en los métodos para determinar su anatomía y su función. Es probable que la definición de función deba incluir parámetros diversos, como geometría, hipertrofia, dilatación, contracción, aporte de oxígeno a la pared y volumen eyectado. En la presente revisión describiremos el comportamiento del VD frente a diversas patologías. En primer lugar analizaremos su comportamiento frente a patologías respiratorias y luego frente a patologías que comprometen la circulación pulmonar y la Insuficiencia cardíaca congestiva (ICC). Nuestro propósito es estimular a la comunidad médica de Enfermedades Respiratorias y Cardiología a profundizar en esta entidad común. La descripción completa de cada uno de los cuadros que causan falla cardíaca derecha, sobrepasa los límites de este artículo.

Palabras clave: Ventrículo derecho, insuficiencia ventricular derecha, cor pulmonale.

\section{Introducción}

El ventrículo derecho (VD) tolera la sobrecarga de volumen mejor que la sobrecarga de presión y tiene buena capacidad de adaptación. Por ejemplo, la insuficiencia tricuspídea es bien tolerada por períodos prolongados, así como la comunicación interauricular, en que la lesión puede permanecer asintomática durante la fase de alto volumen hasta que se desarrollen cambios estructurales en la circulación pulmonar y el shunt se revierta. Aún después de establecida la fisiología de Eisenmenger, el acondicionamiento que significa la sobrecarga de volumen previa,

* Servicio y Laboratorio Broncopulmonar, Clínica Alemana de Santiago. Facultad de Medicina Clínica Alemana, Universidad del Desarrollo.

** Departamento de Cardiología y Laboratorio de Hemodinamia. Clínica Alemana de Santiago. Servicio Médico Quirúrgico Cardiovascular, Laboratorio de Hemodinamia y Cardiología Intervencional, Instituto Nacional del Tórax. *** Departamento de Cardiología y Departamento de Imágenes.Clínica Alemana de Santiago. 
permite que el VD se adapte mejor que los pacientes con hipertensión pulmonar por fibrosis pulmonar idiopática ${ }^{2,3}$.

La sobrecarga de presión del VD puede ser por hipertensión pulmonar (HP) aguda, crónica o estenosis de la válvula pulmonar. La respuesta adaptativa inicial es hipertrofia miocárdica, seguida por disfunción contráctil progresiva y dilatación, para conservar el volumen sistólico. En la medida en que progresa la alteración en la contractilidad se hace evidente la falla cardíaca derecha, caracterizada por aumento de las presiones de llene, disfunción diastólica y disminución del débito. El aumento del tamaño y la sobrecarga de presión del VD finalmente produce disfunción diastólica del ventrículo izquierdo $(\mathrm{VI})^{2,3}$ (Figura 1).
Los mecanismos específicos que llevan a la falla del VD no están claros. Por ejemplo, se postula que se desarrolla isquemia miocárdica, disfunción endotelial y apoptosis de miocitos. En la HP severa terminal, la silueta del VD cambia de configuración y la tensión y el grosor de la pared libre del VD parecen estar inversamente relacionados. Tampoco está claro el mecanismo por el cual un VD dilatado y aparentemente terminal, se recupera después de un trasplante pulmonar. La Figura 2 describe los mecanismos generales de la falla ventricular derecha ${ }^{2-4}$.

Describiremos el comportamiento del VD frente a diferentes entidades patológicas, dividiéndolo en dos partes: primero revisaremos su comportamiento en patologías respiratorias y posteriormente analizaremos su comportamiento
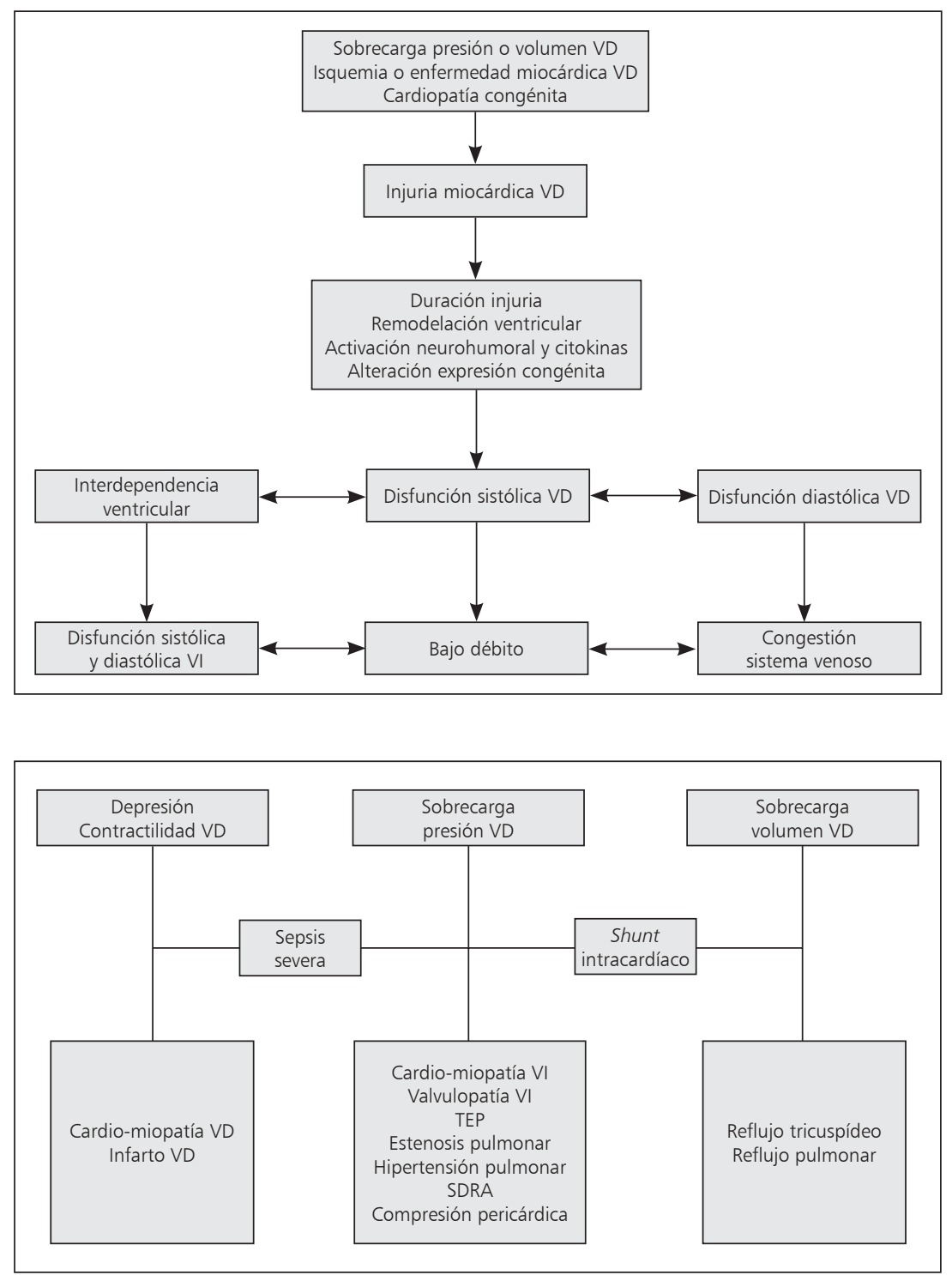

82
Figura 1. Secuencia de mecanismos que comprometen la función del ventrículo derecho y posteriormente la función del ventrículo izquierdo. VD: ventrículo derecho. VI: ventrículo izquierdo. Modificado de Haddad F. et $\mathrm{al}^{3}$.

Figura 2. Mecanismos fisiopatológicos asociados a falla ventricular derecha. VD: ventrículo derecho.VI: ventrículo izquierdo.TEP: Tromboembolismo pulmonar. SDRA: Síndrome de distrés respiratorio agudo. Modificado de Piazza G.et al9 . 


\begin{tabular}{|c|}
\hline Sobrecarga de presión \\
\hline Falla cardíaca izquierda \\
\hline Embolía pulmonar \\
\hline Otras causas de hipertensión pulmonar \\
\hline Obstrucción del tracto de salida VD \\
\hline Estenosis pulmonar \\
\hline Sobrecarga de volumen \\
\hline Reflujo tricuspídeo \\
\hline Reflujo pulmonar \\
\hline Comunicación interauricular \\
\hline Drenaje venoso anómalo \\
\hline Fístula coronaria a $\mathrm{AD}$ o VD \\
\hline Isquemia e infarto \\
\hline Infarto del VD \\
\hline $\begin{array}{l}\text { Contribución de isquemia a disfunción VD por } \\
\text { sobrecarga de presión o volumen }\end{array}$ \\
\hline Enfermedades del miocardio \\
\hline Miocardiopatías \\
\hline Displasia arritmogénica del VD \\
\hline Limitación del llenado de VD \\
\hline Estenosis tricuspídea \\
\hline Estenosis de vena cava superior \\
\hline Cardiopatías congénitas complejas \\
\hline Anomalía de Ebstein \\
\hline Tetralogía de Fallot \\
\hline Transposición de los grandes vasos \\
\hline Enfermedades del pericardio \\
\hline Pericarditis constrictiva \\
\hline
\end{tabular}

Figura 3. Causas de falla cardíaca derecha. AD: aurícula derecha. VD: ventrículo derecho. Modificado de Haddad F. et $\mathrm{al}^{3}$.

en patologías que comprometen la circulación pulmonar propiamente tal y en insuficiencia cardíaca congestiva (ICC) (Figura 3 ).

\section{Corazón pulmonar en las enfermedades respiratorias}

\section{Fisiopatología}

El corazón pulmonar (CP) corresponde a falla del VD por enfermedades que afectan la función y/o estructura de los pulmones, excepto cuando son el resultado de enfermedades que afectan el lado izquierdo del corazón. Los pacientes con corazón pulmonar pueden presentar hipertrofia ventricular derecha, disfunción asintomática o falla cardíaca derecha. Entre 10 a $30 \%$ de las insuficiencias cardíacas descompensadas que se hospitalizan corresponden a CP, siendo la causa más común la enfermedad pulmonar obstructiva crónica (EPOC). Otras causas capaces de causar $\mathrm{CP}$ son las enfermedades pulmonares insterticiales, las alteraciones ventilatorias restrictivas y las alteraciones del control ventilatorio ${ }^{3,6}$.

La HP en las enfermedades respiratorias hipoxémicas es el resultado de factores múltiples, que incluyen vasoconstricción hipóxica, hipercarbia, acidemia, distorsión de los vasos pulmonares, aumento del débito cardíaco y policitemia. La remodelación vascular en respuesta a la hipoxia, está determinada por una serie de factores que incluyen óxido nítrico, endotelina, serotonina, factor-1 inducible por hipoxia y los mediadores de la inflamación. La acidosis aumenta la resistencia vascular pulmonar y potencia el efecto de la hipoxia. Finalmente, los cambios estructurales como proliferación de la íntima e hipertrofia de la musculatura lisa, hacen manifiesta la $\mathrm{HP}^{3,6,7}$.

El diagnóstico de HP requiere de cateterización cardíaca derecha, con una presión media de arteria pulmonar $\geq 25 \mathrm{mmHg}$, una presión de enclavamiento capilar pulmonar $\leq 15 \mathrm{mmHg}$ y una resistencia vascular pulmonar de 3 unidades Woods. La interpretación es difícil en pacientes con patología pulmonar crónica y disfunción diastólica del VI, asociación bastante común. En los pacientes con HP significativa se debe buscar enfermedad tromboembólica crónica, considerando su potencial tratamiento y su incidencia aumentada en pacientes con EPOC y fibrosis pulmonar idiopática ${ }^{6-9}$.

El VD es una cámara delgada, distensible, y de baja presión. La arteria coronaria derecha aporta flujo de sangre a la pared libre del VD en sístole y diástole, protegiendo al VD de la isquemia. Cuando la sobrecarga de presión es crónica, el VD se hipertrofia y finalmente se dilata, lo que causa disfunción sistólica y diastólica (Figura 4). En el VD también puede haber isquemia, por flujo insuficiente de la arteria coronaria a un VD hipertrofiado, y reducción del gradiente de presión entre la coronaria derecha y la cavidad ventricular derecha en sístole y diástole. En los pacientes con enfermedades pulmonares obstructivas, la hiperinsuflación disminuye el retorno venoso y reduce el llene del VD. En las enfermedades pulmonares restrictivas, la incapacidad de la caja torácica en distenderse es otra causa que deteriora el llene cardíaco ${ }^{1-6}$.

El grado de disfunción ventricular izquierda secundario a disfunción ventricular derecha es motivo de debate. En una revisión retrospectiva 

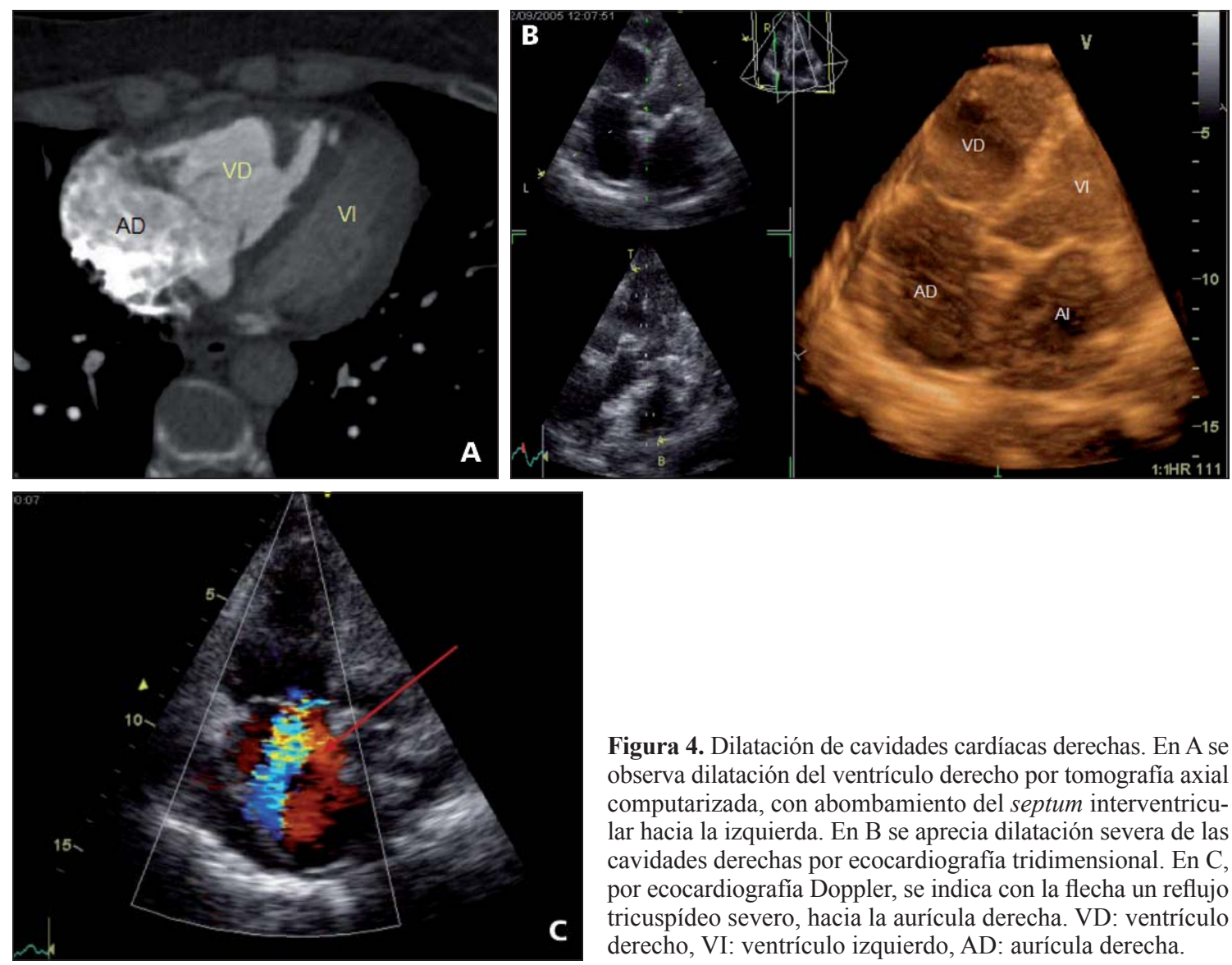

Figura 4. Dilatación de cavidades cardíacas derechas. En A se observa dilatación del ventrículo derecho por tomografía axial computarizada, con abombamiento del septum interventricular hacia la izquierda. En B se aprecia dilatación severa de las cavidades derechas por ecocardiografía tridimensional. En C, por ecocardiografía Doppler, se indica con la flecha un reflujo tricuspídeo severo, hacia la aurícula derecha. VD: ventrículo derecho, VI: ventrículo izquierdo, AD: aurícula derecha.

de 434 pacientes con enfermedad pulmonar terminal, incluyendo EPOC, enfermedades pulmonares intersticiales, HP primaria y Eisenmenger, la prevalencia de disfunción ventricular derecha (fracción de eyección del VD $<45 \%$ ), fue del $66 \%$ y la disfunción ventricular izquierda (fracción de eyección del VI $<45 \%$ ), del $6,4 \%$ de los pacientes. El VD y el VI comparten el septum y el saco pericárdico, por lo que un ventrículo influencia el otro. La capacidad limitada del pericardio de distenderse significa que un cambio significativo en el volumen del VD limita el volumen del VI por desplazamiento del septum hacia la izquierda. Los cambios acentuados de presión intrapleural de los pacientes con enfermedades respiratorias contribuyen a aumentar la presión de arteria pulmonar y modifican el retorno venoso hacia el VD. La dilatación del VD causa disfunción diastólica del VI, aumenta su presión de fin de diástole y reduce el volumen sistólico ${ }^{6-9}$.

\section{Presentación clínica}

La HP en enfermedades respiratorias hipoxémicas es común, pero habitualmente es leve a moderada. La disnea es síntoma habitual, pero debido a su inespecificidad (por estar presente en la enfermedad de base), no aporta mayormente en el diagnóstico. Un cambio en la disnea, dolor torácico o edema de extremidades inferiores sugiere HP. Síntomas habituales de CP son edemas, sobrecarga venosa, disminución del débito, intolerancia al ejercicio y arritmias. Los signos clínicos como crecimiento del VD, reforzamiento del segundo ruido, reflujo tricuspídeo y R4 derecho, suelen estar enmascarados por la signología pulmonar $^{8-11}$.

Varios indicadores electrocardiográficos sugieren CP: desviación a la derecha del eje eléctrico, onda P pulmonar, patrón $\mathrm{S}_{1} \mathrm{~S}_{2} \mathrm{~S}_{3}, \mathrm{~S}_{1} \mathrm{Q}_{3}$, hipertrofia ventricular derecha y bloqueo de rama derecha. El bajo voltaje QRS, es más frecuente en el $\mathrm{CP}$ asociado con EPOC que con otras enfermedades pulmonares. Sin embargo, los cambios ECG no son sensibles. En una serie de pacientes con EPOC, sólo el 33\% de aquellos que tenían aumento de la resistencia vascular pulmonar presentaba signos ECG de CP. La radiografía de tórax puede demostrar cardiomegalia, ensanchamiento 
de las arterias pulmonares y disminución del aire retroesternal ${ }^{8-11}$.

A pesar que el ecocardiograma es útil en el CP, frecuentemente hay "mala ventana acústica" por la patología pulmonar. Por otro lado, si bien es un método sensible ( $85 \%)$, su especificidad es sólo del $55 \%$, con sobre clasificación de $\mathrm{CP}$ en la mitad de los pacientes. Sin embargo, considerando la baja prevalencia de $\mathrm{CP}$, un ecocardiograma sin alteraciones tiene un valor predictivo negativo elevado (sobre 98\%), en consecuencia, un ecocardiograma normal prácticamente descarta $\mathrm{CP}$, pero si está alterado, su resultado debe ser interpretado con cautela ${ }^{12-17}$.

El péptido natriurético (BNP) producido por miocitos auriculares y ventriculares, es útil en la búsqueda de ICC asociada. En pacientes con EPOC y $\mathrm{BNP}<100 \mathrm{pg} / \mathrm{ml}$, prácticamente se descarta ICC, así como un BNP superior a 500 $\mathrm{pg} / \mathrm{ml}$, plantea con alta probabilidad la presencia de $\operatorname{ICC}^{6,10,17,18}$.

\section{Enfermedad pulmonar obstructiva crónica}

La EPOC es la causa más común de CP, sin embargo, el porcentaje que desarrolla $\mathrm{CP}$ no es conocido. En estos pacientes las presiones de arteria pulmonar suelen estar levemente elevadas y el desarrollo de CP está relacionado con la severidad de la EPOC, el deterioro de la capacidad de difusión, el grado de hipoxemia y la vasoconstricción pulmonar hipóxica. La presencia de HP es indicador de mal pronóstico, aumenta la frecuencia de hospitalizaciones y se asocia con peor sobrevida ${ }^{6-8,11}$.

La presencia de HP se ha evaluado en estudios longitudinales. En 131 pacientes con EPOC estudiados con cateterización cardíaca derecha (efectuada en dos oportunidades separadas por 6,8 años), en la evaluación inicial ningún paciente presentaba HP en reposo, pero el $76 \%$ la presentaba en ejercicio. En la segunda evaluación, el $25 \%$ de los pacientes presentaba HP en reposo (de ellos, la mayoría presentaba HP en ejercicio previamente).

Los estudios varían según la población y la metodología utilizada. Parte de la variabilidad se debe a la utilización de ecocardiografía versus cateterización cardíaca derecha. En un estudio reciente, Burgess y cols ${ }^{5}$, demostraron que el índice de diámetro de fin de diástole del VD y la velocidad de llenado tardío, eran factores independientes de sobrevida. Otros factores pronósticos en EPOC incluyen la severidad del defecto ventilatorio obstructivo y las comorbilidades asociadas ${ }^{6-8,11,19,20}$.
El oxígeno es la única terapia en EPOC que ha demostrado mejorar la HP, el CP y la sobrevida. En el estudio del British Medical Research Council y en el estudio NOTT se demostró que aquellos pacientes que no eran tratados con oxígenoterapia nocturna presentaban mayor resistencia vascular pulmonar, la que disminuyó en forma marcada en aquellos pacientes que usaban $\mathrm{O}_{2}$ en forma continua. Otros estudios han demostrado que el uso de $\mathrm{O}_{2}$ por períodos prolongados, se asocia a disminución discreta de las presiones de arteria pulmonar, efecto que se pierde con su suspensión - $^{6-9,11,19,20}$.

El uso de vasodilatadores en EPOC para disminuír la HP ha sido poco estudiado. El Sildenafil ha demostrado disminuír las presiones de arteria pulmonar en estudios restringidos, al igual que el Nifedipino, sin embargo este último se ha asociado a deterioro en la oxigenación. El Losartan por otro lado, no ha demostrado resultados consistentes $3,4,6,7,20,21$.

\section{Enfermedades pulmonares intersticiales}

Las enfermedades insterticiales se han asociado también a CP. La prevalencia de HP en esta patología varía significativamente. Estudios recientes demuestran amplios rangos de incidencia, que varían entre 8 y $84 \%$. Al igual que en EPOC, los criterios y la modalidad diagnóstica utilizada varían ampliamente ${ }^{5,6,7,20}$.

En la evaluación de pacientes candidatos a trasplante pulmonar, la incidencia es elevada, variando entre 31,6 a 73,8\% según la serie. $\mathrm{La}$ HP en fibrosis pulmonar idiopática es una característica per se, al dañar y obliterar la vasculatura pulmonar, con trombosis in situ y organización fibrosa ${ }^{6,7,20}$.

Un estudio ha postulado que hipoxemia $\left(\mathrm{SaO}_{2}\right.$ $\leq 88 \%)$ o hipercapnia $\left(\mathrm{PCO}_{2} \geq 55 \mathrm{mmHg}\right)$, combinado con una capacidad de difusión con monóxido de carbono (DLCO) $\leq 40 \%$, indica la presencia de hipertensión pulmonar, con una valor predictivo positivo de $87 \%$ y valor predictivo negativo de $82 \%$. Otro estudio demostró una correlación discreta entre la DLCO e HP; en el $40 \%$ de ellos había HP, sin embargo, si la DLCO era $\leq 30 \%$ se duplicaba la prevalencia. En consecuencia, se debe sospechar HP en pacientes con fibrosis pulmonar idiopática, hipoxemia de reposo y baja DLCO $^{6,7,20}$.

Existen datos limitados respecto al uso de vasodilatadores en fibrosis pulmonar idiopática, los que teóricamente pueden empeorar la relación ventilació/perfusión y aumentar la hipoxemia. Tanto el Sildenafil como el Epoprosternol demostraron disminuir la HP en 16 pacientes, sin 
embargo, el Sildenafil mejoró la hipoxemia y el Epoprosterenol la empeoró. Otros estudios han demostrado mejoría en el test de caminata en 6 minutos con Sildenafil $6,7,10,20,21$.

\section{Enfermedades ventilatorias neuromusculares}

Las enfermedades ventilatorias neuromusculares como las distrofias musculares, esclerosis lateral amiotrófica, miastenia gravis, y aquellas que alteran la expansión de la pared del tórax, como cifoescoliosis y obesidad mórbida, funcionalmente disminuyen la distensibilidad tóracopulmonar y los volúmenes pulmonares, causando hipoventilación alveolar, hipercapnia e hipoxemia. Esta categoría corresponde a patologías diversas, por lo que la prevalencia de CP es desconocida ${ }^{4,7,10,20}$.

La hipoventilación nocturna se desarrolla antes que sea evidente la insuficiencia respiratoria durante el día. La presión de arteria pulmonar aumenta en relación a la hipoventilación durante el sueño, y la severidad de la hipoventilación nocturna se correlaciona con la presencia de CP. Una serie de publicaciones ha demostrado que la ventilación mecánica no invasiva puede mejorar la HP, y parcialmente revertir el CP en estos pacientes s,7,10,20. $^{4}$.

\section{Alteraciones del control ventilatorio}

Este grupo de patologías incluye el Síndrome de apneas de sueño y el Síndrome obesidad asociada a hipoventilación. Estos cuadros de hipoventilación nocturna se acompañan además de la hipercapnia, de hipoxemia, y conducen a HP en los casos más severos ${ }^{1,4,6,10,20}$.

En un estudio observacional de 50 pacientes con sídrome de apnea-hipopnea obstructiva del sueño (SAHOS), aquellos pacientes que tenían $\mathrm{CP}$, presentaban hipoxemia más profunda durante la noche y durante el día. Aquellos pacientes que no presentaban hipoxemia durante el día no tenían CP. A pesar de esto, múltiples estudios no han logrado detectar relación entre el Síndrome de Apneas de sueño, hipertensión pulmonar severa y falla del $\mathrm{VD}^{1,4,6,10,20}$.

\section{Patologías de la circulación pulmonar e insuficiencia cardíaca congestiva}

\section{Tromboembolismo pulmonar}

La causa más frecuente de sobrecarga aguda de presión en el VD es el Tromboembolismo pulmonar (TEP), por obstrucción trombótica de arterias pulmonares principales, lobares o segmentarias, y continúa siendo causa importante de morbilidad y mortalidad. La mortalidad del TEP está estrechamente relacionada con el grado de falla ventricular derecha y la inestabilidad hemodinámica ${ }^{22,24}$.

El VD no es capaz de generar presiones medias de arteria pulmonar $>40 \mathrm{mmHg}$ en sobrecargas agudas de presión, por esta razón frente a un TEP masivo, la falla cardíaca es precoz. En este escenario la interdependencia ventricular es determinante en la falla cardíaca derecha; la dilatación del VD desvía el septum hacia la izquierda, modifica la geometría del VI y causa compresión pericárdica. Estas alteraciones disminuyen la distensibilidad y la precarga del VI, reduciendo progresivamente el débito cardíaco. En este contexto los pacientes se pueden dividir en 3 grupos: 1) Hemodinámicamente estables, (mortalidad $<4 \%$ ); 2) Con disfunción VD pero sin shock, (mortalidad 5-15\%) y 3 ) Con shock cardiogénico, (mortalidad 20- 50\%) $)^{3,22,24}$.

Entre los pacientes que presentan TEP, menos del 5\% desarrolla enfermedad tromboembólica crónica, por otro lado, más del $60 \%$ de ellos no tiene antecedente de TEP previo. La trombosis in situ parece jugar un papel importante en la fisiopatología del TEP crónico; comparado con los pacientes con HP primaria, los pacientes con TEP crónico tienden a presentar menor débito cardíaco para el mismo nivel de presiones de arteria pulmonar ${ }^{3,25,29}$.

\section{Hipertensión arterial pulmonar}

En la hipertensión arterial pulmonar, la sobrecarga de presión crónica sobre el VD presenta cambios histológicos, caracterizados por aumento en la densidad del tejido conectivo miocárdico, además en la hipertrofia ventricular derecha, se producen cambios en la expresión genética, con recapitulación del patrón genético del corazón fetal. Por otro lado, algunos pacientes con HP desarrollan falla ventricular derecha antes que otros, lo que supone diferencias fenotípicas. De hecho, algunos pacientes desarrollan mayor hipertrofia ventricular derecha que otros, para el mismo grado de $\mathrm{HP}^{30-32}$.

Los pacientes con HP primaria presentan altos niveles de catecolaminas, lo que afecta adversamente la remodelación ventricular. $\mathrm{La}$ estimulación adrenérgica excesiva se asocia a mayor resistencia vascular pulmonar y menor índice cardíaco. De hecho, la magnitud de esta respuesta constituye un factor pronóstico en la falla cardíaca derecha. La sobrecarga de presión del VD está asociada a pérdida de la respuesta al efecto inotrópico de la angiotensina II y a desacoplamiento de los receptores de la angiotensina I. En pacientes con HP primaria y falla cardíaca derecha, la activación del sistema endotelina, con 
niveles elevados de endotelina I, se asocia con mayor severidad de la falla del $\mathrm{VD}^{31-33}$.

La disminución de la capacidad de ejercicio, es el factor pronóstico de mortalidad más importante en pacientes con falla del VD asociada a HP primaria, tanto en el test de caminata de 6 minutos como en la disminución del consumo de $\mathrm{O}_{2}$ máximo bajo $10 \mathrm{ml} / \mathrm{kg} / \mathrm{min}$. Otros factores pronósticos incluyen dilatación y disfunción sistólica del VD en la ecocardiografía, descenso del índice cardíaco bajo $2 \mathrm{~L} / \mathrm{min} / \mathrm{m}^{2}$, aumento de la presión de aurícula derecha sobre $20 \mathrm{mmHg}$, incompetencia cronótropa y arritmias ${ }^{32-34}$.

\section{Insuficiencia cardíaca izquierda}

La causa más común de hipertensión pulmonar crónica es la asociada con la falla ventricular izquierda. La falla ventricular derecha se asocia a la falla ventricular izquierda por múltiples mecanismos: la falla del VI aumenta la post carga del VD, aumenta las presiones venosas y finalmente la presión arterial pulmonar. Cuando el VD falla en el contexto de una insuficiencia ventricular izquierda, es incapaz de sostener la precarga del VI. Además, el mismo proceso cardiomiopático o la isquemia miocárdica, pueden afectar ambos ventrículos ${ }^{4,35-37}$.

La disfunción ventricular izquierda disminuye la perfusión de la arteria coronaria derecha, constituyéndose en un determinante de la función ventricular derecha. La dilatación ventricular en un compartimento pericárdico limitado, restringe la función diastólica del VD, y el septum, que es el componente fundamental de la interdependencia ventricular, compromete la función de ambos ventrículos. En forma inversa, la sobrecarga de presión del VD, como sucede en la HP, compromete la función ventricular izquierda ${ }^{38-40}$.

Las diversas influencias que afectan la función del VD por falla ventricular izquierda, sugieren que el estado del VD constituye una vía final en la evolución de la ICC, por lo que es un indicador de mal pronóstico. Por otro lado, cuando la función del VD está preservada en pacientes con ICC e HP, es una ventaja en la expectativa de vida ${ }^{38-41}$.

Varios índices ecocardiográficos de función ventricular derecha son indicadores de mal pronóstico en ICC, entre ellos la fracción de acortamiento del VD (FAVD) en pacientes con miocardiopatía o cardiopatía isquémica. La FAVD es el único parámetro independiente en análisis multivariados que incluyen fracción de eyección del VI (FEVI), índice cardíaco, y resistencia vascular pulmonar. La fracción de eyección del VD (FEVD) se correlaciona mejor con la capacidad de ejercicio que la FEVI. De hecho, en pacientes con ICC moderada, la FEVD es un predictor independiente de sobrevida, al igual que el consumo de $\mathrm{O}_{2}$ máximo $35,38-42$.

La morfología especial del VD dificulta la evaluación de su volumen y el cálculo de la FEVD. La excursión sistólica del plano valvular tricuspídeo se correlaciona bien con la FEVD. Con Doppler tisular se estima la onda $S$ del anillo tricuspídeo, con buena sensibilidad para diferenciar disminución significativa de la FEVD. El índice de performance (o desempeño) del VD relaciona los tiempos de llene tricuspídeo y flujo expulsivo pulmonar, entregando un índice global de la función sistólica y diastólica del VD. Estos indicadores tienen un valor predictivo negativo superior al $90 \%$ para descartar disfunción del $\mathrm{VD}^{1-3,12-15}$.

\section{Infarto del ventrículo derecho}

El VD demuestra una capacidad sobresaliente de recuperar función sistólica en el infarto agudo del VD, demostrando su resistencia al daño isquémico irreversible. Sin embargo, un infarto extenso del VD puede causar suficiente daño miocárdico como para causar insuficiencia cardíaca, shock y muerte, en ausencia de daño ventricular izquierdo, sobrecarga de volumen o de presión preexistentes.

La falla hemodinámica derecha bajo estas circunstancias, sugiere que un VD dilatado e hipocontráctil juega un papel activo en comprometer todo el status hemodinámico. No está establecido si esto se debe a interdependencia ventricular, compromiso septal u otro mecanismo. La reperfusión precoz por angioplastía, supone un quiebre en la mala evolución de la falla circulatoria por compromiso del $\mathrm{VD}^{3,4,9,10,43,44}$.

\section{Cardiopatías congénitas}

En el Síndrome de Eisenmenger y en la estenosis pulmonar congénita, la falla cardíaca derecha es tardía al compararlos con otras causas de HP. El Síndrome de Eisenmenger tiene una sobrevida de $80 \%$ a 10 años y de $42 \%$ a 25 años. Se postula que la resistencia del VD a la sobrecarga crónica de presión, se debe a la preservación del fenotipo fetal, con el mismo grosor de la pared del VD y del VI a lo largo de la vida, y por la presencia de un cortocircuito intracardíaco alternativo (comunicación interauricular, interventricular, o ductus persistente). El aumento del débito por el shunt, aún a costa de desaturación sistémica, descarga el VD postergando su dilatación. Este mecanismo ha sido imitado en el tratamiento de pacientes con HP primaria severa, al realizar septostomía auricular ${ }^{3,4,6,10}$. 
En la estenosis pulmonar congénita, el grado de hipertrofia ventricular derecha varía dependiendo de la severidad de la obstrucción. El VD habitualmente se adapta bien a este tipo de estenosis, presentando tardíamente falla cardíaca derecha y regurgitación tricuspídea. Del mismo modo, la corrección de la estenosis pulmonar por valvulotomía quirúrgica o percutánea, conduce a una rápida remodelación ventricular derecha $a^{3,4,6,10}$.

En pacientes con tetralogía de Fallot, la reparación de la estenosis del tracto de salida del VD conduce a insuficiencia valvular pulmonar progresiva, deterioro de la función del VD y arritmias ventriculares. La determinación de los volúmenes del VD, de la FEVD y de la regurgitación pulmonar, tanto por ecocardiografía como por resonancia nuclear magnética, son relevantes en la definición de una nueva reparación quirúrgica ${ }^{3,4,6,10,45}$.

La transposición de los grandes vasos es una situación particular en las cardiopatías congénitas. El VD morfológico está situado a la izquierda y conectado a la aorta, y el VI morfológico está a la derecha y conectado a la arteria pulmonar. La evolución natural del VD sometido a la presión sistémica, es insuficiencia de la válvula aurículoventricular (tricúspide en este caso), y falla de este ventrículo. Las opciones de tratamiento son muy limitadas; la estrechez calibrada de la arteria pulmonar adapta el ventrículo pulmonar antes de la cirugía correctora, evita el desplazamiento del septum y retrasa la falla del VD sistémico. A veces, la única opción es el trasplante cardía$\mathrm{co}^{3,4,6,10,46}$.

\section{Líneas de tratamiento de la falla ventricular derecha}

Los tratamientos actuales están dirigidos hacia la vasoconstricción pulmonar, la proliferación celular y los factores trombóticos. Estos han mejorado la calidad de vida y la sobrevida en muchos pacientes con HP severa. Por otro lado, terapias que reducen la post carga, los $\beta$ bloqueadores, los inótropos y los diuréticos, han mejorado el estado funcional y el pronóstico de los pacientes con falla ventricular izquierda ${ }^{3-7,10,18,47-49}$.

La infusión crónica de Prostaciclina (Epoprosterenol), en pacientes con hipertensión arterial pulmonar parece mejorar la función del VD, a pesar de que la hipertrofia puede persistir después de un año de observación. El antagonista del receptor de endotelina (Bosentan), también ha demostrado mejoría en los parámetros ecocardiográficos de función del VD. Sin embargo, los efectos miocárdicos específicos de estas terapias para la hipertensión pulmonar no están totalmente $\operatorname{aclarados}^{48-52}$.
La función ventricular derecha, la interdependencia ventricular y el acoplamiento del VD al sistema arterial pulmonar, están siendo ampliamente evaluados como potenciales objetivos de terapia $^{48-54}$.

\section{Epílogo}

Hemos descrito el comportamiento del VD frente a diversas patologías, relacionándolo con enfermedades respiratorias, enfermedades que comprometen la circulación pulmonar propiamente tal e insuficiencia ventricular izquierda. La unidad aparato respiratorio, circulación pulmonar, VD y VI forma parte de nuestra práctica habitual. Suponemos un amplio desarrollo conceptual y terapéutico en este aspecto, y vislumbramos la necesidad de un trabajo colaborativo entre especialistas en Enfermedades Respiratorias y Cardiólogos, para enfrentar estas patologías.

\section{Bibliografía}

1.- CAVIEDES I, URIARTE P. Fisiopatología del ventrículo derecho. Rev Chil Enf Respir 2009; 25: 170-181.

2.- HADDAD F, HUNT S A, ROSENTHAL D N, MURPHY D J. Right ventricular function in cardiovascular disease, part I. Anatomy, Physiology, aging, and functional assessment of the right ventricle. Circulation 2008; 117: 1436-48.

3.- HADDAD F, DOYLE R, MURPHY D J, HUNT S A. Right ventricular function in cardiovascular disease, part II. Pathophysiology, clinical importance, and management of right ventricular failure. Circulation 2008; 117 : 1717-31.

4.- VOELKEL N, QUAIFE R A, LEINWAND L A, BARST R J, MC GOON M D, MELDRUM D R, et al. Right ventricular function and failure. Report of a National Hearth, Lung and Blood Institute working group on cellular and molecular mechanisms of right heart failure. Circulation 2006; 114: 1883-91.

5.- HAN M K, MC LAUGHLIN V V, CRINER G J, MARTÍNEZ F J. Pulmonary diseases and the hearth. Circulation 2007; 116: 2992-3005.

6.- WRIGHT J L, LEVY R D, CHURG A. Pulmonary hypertension in chronic obstructive pulmonary disease: current theories of pathogenesis and their implications for treatment. Thorax 2005; 60: 605-9.

7.- TRABUT G, DAURIAT G, STERN JB, LOGEART D, LEVY A, MARRASH-CHALA R, et al. Pulmonary hemodynamics in advanced COPD candidates for lung volume reduction surgery or lung transplantation. Chest 2005; 127: 1531-6.

8.- BLEEKER G B, STEENDIJK P, HOLMAN E R, YU 
C M, BREITHARDT O A, KAANDORP T A, et al. Acquired right ventricular dysfunction. Hearth 2006; 92 (suppl 1) i14-8.

9.- PIAZZA G, GOLDHABER S. The acutely decompensated right ventricle. Pathways for diagnosis and management. Chest 2005; 128: 1836-52.

10.- CHAOUAT A, BUGNET AS, KADAOUI N, SCHOTT R, ENACHE L, DUCOLONE A, et al. Severe pulmonary hypertension and chronic obstructive pulmonary disease. Am J Respir Crit Care Med 2005; 172: 189-94.

11.- FISHER M R, CRINER G J, FISHMAN A P, HASSOUN P M, MINAI O A, SCHARF S M, et al. Estimating pulmonary artery pressures by echocardiography in patients with emphysema. Eur Respir J 2007; 30: 914-21.

12.- BLEEKER G B, STEENDIJK P, HOLMAN E R, YU C M, BREITHARDT OA, KAANDORP T A, et al. Assesing right ventricular function: the role of echocardiography and complementary technologies. Hearth 2006; 92 (suppl 1): i 19-26.

13.- HO S Y, NIHOYANNOPOULOS P. Anatomy, echocardiography, and normal right ventricular dimensions. Hearth 2006; 92 (suppl 1): i2-13.

14.- FISHER M R, FORFIA P R, CHAMERA E, HOUSTEN-HARRIS T, CHAMPION H C, GIRGIS R E, et al. Accuracy of Doppler echocardiography in the hemodynamic assessment of pulmonary hypertension. Am J Resp Crit Care Med 2009; 179: 615-21.

15.- JIMÉNEZ D. Valor pronóstico de la Ecocardiografia transtorácica en pacientes estables hemodinámicamente con Tromboembolía de pulmón aguda sintomática. Arch Bronconeumol 2007; 43: 490-4.

16.- NAGAYA N, NISHIKIMI T, UEMATSU M, SATOH T, KYOTANI S, SAKAMAKI F, et al. Plasma brain natriuretic peptide as a prognostic indicator in patients with primary pulmonary hypertension. Circulation 2000; 102; 865-70.

17.- KUCHER N, PRINTZEN G, GOLDHABER S Z. Prognostic role of natriuretic peptide in acute pulmonary embolism. Circulation 2003; 107: 2545- 7.

18.- PEINADO V I, PIZARRO S, BARBERÁ J A. Pulmonary vascular involvement in COPD. Chest 2008; 134: 808-14.

19.- GALIE N, HOEPER M, HUMBERT M, TORBICKI A, VACHIERY J L, BARBERA J A, et al. Guidelines for the diagnosis and treatment of pulmonary hypertension. The Task Force for the Diagnosis and Treatment of Pulmonary Hypertension of the European Society of Cardiology (ESC) and the European Respiratory Society (ERS) endorsed by the International Society of Heart and Lung Transplantation (ISHLT). Eur Respir J 2009; 34: $1219-63$

20.- COLLARD H R, ANSTROM K J, SCHWARZ M I, ZISMAN DA. Sildenafil improves walk distance in idiopathic pulmonary fibrosis. Chest 2007; 131: 897-9.

21.- TORBICKI A, PERRIER A, KONSTANTINIDES
S, AGNELLI G, GALIĖ N, PRUSZCZYK P, et al. Guidelines on the diagnosis and management of acute pulmonary embolism: The Task Force for the Diagnosis and Management of Acute Pulmonary Embolism of the European Society of Cardiology (ESC). Eur Heart J 2008; 29: 2276-315.

22.- GOLDHABER S Z, ELLIOTT C G. Acute pulmonary embolism: part I. epidemiology, pathophysiology, and diagnosis. Circulation 2003; 108: 2726-9.

23.- GOLDHABER S Z, VISANI L, DE ROSA M. Acute pulmonary embolism: clinical outcomes, in the internacional cooperative pulmonary embolism registry (ICOPER). Lancet 1999; 353: 1386-9.

24.- PENGO V, LENSING A W, PRINS M H, MARCHIORI A, DAVIDSON B L, TIOZZO F, et al Incidence of chronic thromboembolic pulmonary hypertension after pulmonary embolism. N Engl J Med 2004; 350: 2257 64.

25.- HOEPER M M, MAYER E, SIMMONEAU G, RUBIN L J. Chronic thromboembolic pulmonary hypertension. Circulation 2006; 113: 2011-20.

26.- CONDLIFFE R, KIELY D G, GIBBS J S, CORRIS P A, PEACOCK A J, JENKINS D P, et al. Improved outcomes in medically and surgically treated chronic thromboembolic pulmonary hypertension patients. Am J Respir Crit Care Med 2008; 177: 1122 -7.

27.- FEDULLO P F, AUGER W R, KERR K M, RUBIN LJ, et al. Chronic thromboembolic pulmonary hypertension. N Engl J Med 2001; 345: 1465-72.

28.- LANG I M. Chronic thromboembolic pulmonary hypertension-not so rare after all. N Engl J Med 2004; 350 : 2236-8.

29.- SIMONNEAU G, ROBBINS I, BEGHETTI M, et al. Updated clinical classification of pulmonary hypertension. J Am Coll Cardiol 2009; 54: Suppl. 1, S43-S54.

30.- MCLAUGHLIN V V, MCGOON M D. Pulmonary arterial hypertension. Circulation 2006; 114: 1417-31.

31.- BOGAARD H J, ABE K, VONK NOORDEGRAAF A, VOELKEL N. The Right Ventricle Under Pressure. Cellular and Molecular Mechanisms of Right-Heart Failure in Pulmonary Hypertension Chest 2009; 135: 794-804

32.- BRISTOW M R, ZISMAN L S, LOWES B D, ABRAHAM W T, BADESCH D B, GROVES B M, et al. The pressure overload right ventricle in pulmonary hypertension. Chest 1998; 114; 101S-6S.

33.- WENSEL R, OPITZ CF, ANKER S D, WINKLER J, HOFFKEN G, KLEBER FX, et al. Assessment of survival in patients with primary pulmonary hypertension. Importance of cardiopulmonary exercise testing. Circulation 2002; 106: 319-24.

34.- CHAMPION H C, MICHELAKIS E D, HASSOUN P M. Comprehensive Invasive and Noninvasive Approach to the Right Ventricle-Pulmonary Circulation Unit. State of the Art and Clinical and Research Implications. Circulation 2009;120: 992-1007.

35.- CECCONI M, JOHNSTONE E, RHODES A. What role 
does the right side of the heart play in circulation?. Crit Care 2006; 10 suppl 3: S5.

36.- GEHLBACH B K, GEPPERT E. The pulmonary manifestations of left hearth failure. Chest 2004; 125: 669-82.

37.- KLIMA U P, LEE M Y, GUERRERO J L, LARAIA P J, LEVINE R A, VLAHAKES G J. Determinants of maximal right ventricular function: role of septal shift. J Thorac Cardiovasc Surg 2002; 123: 72-80.

38.- SALEH S, LIAKOPOULOS O J, BUCKBERG G D. The septal motor of biventricular function. Eur J Cardiothorac Surg 2006; 29 (suppl 1): S126-38.

39.- BUCKBERG G D. The ventricular septum: the lion of the right ventricular function, and its impact on right ventricular restoration. RESTORE group. Eur J Cardiothorac Surg 2006; 29 (suppl 1): S 272-8.

40.- GHIO S, GAVAZZI A, CAMPANA C, INSERRA C, KLERSY C. Independent and additive prognostic value of right ventricular systolic function and pulmonary artery pressure in patients with chronic hearth failure. J Am Coll Cardiol 2001; 37; 183-8.

41.- MENDES L A, DEE G W, PICARD M H, PALCAIOS I F, NEWELL J, DAVIDOFF R. Right ventricular dysfunction: an independent predictor of adverse outcome in patients with miocarditis. Am Hearth J 1994; 128; 301-7.

42.- DE GROOTE P, MILLAIRE A, FOUCHER HOSSEIN C, NUGUE O, MARCHANDISE X, DUCLOUX G, LABLANCHE J M. Right ventricular Ejection fraction is an independent predictor of survival in patients with moderate heath failure. J Am Coll Cardiol 1998; 32: 948-54.

43.- KLIMA U P, GUERRERO J L, VLAHKES G J. Miocardial perfusion and right ventricular function . An thorac Cardiovasc Surg 1999; 5: 74-80.

44.- METHA S R, EIKELBOOM J W, NATARAJAN M K, DÍAZ R, YI C, GIBBONS R J, et al. Impact of right ventricular involvement on mortality and morbidity in patients with inferior myocardial infarction. J Am Coll Cardiol 2001; 37: 37-43.

45.- GATZOULIS M A, BALAJI S, WEBBER S A, SIU S C, HOKANSON J S, POILE C, et al. Risk factors for arrhythmia and sudden cardiac death late after repair of tetralogy of Fallot: A multicentre study. Lancet 2000;
356: $975-81$.

46.- DUNCAN B, MEE R. Management of the Failing Systemic Right Ventricle Seminars in Thoracic and Cardiovascular Surgery 2005; 17: 160-9.

47.- MEBAZAA A, KARPATI P, RENAUD E, ALGOTSSON L. Acute right ventricular failure. From pathophysiology to new treatments. Intensive Care Med 2004; 30: $185-96$

48.- HANDOKO M L, DE MAN F S, ALLAART C P, PAULUS W J, WESTERHOF N, VONK NOORDEGRAAF A. Perspectives on novel therapeutics strategies for right hearth failure in pulmonary arterial hypertension: lessons from the lesft hearth. Eur Respir Rev 2010; 19: 72-82.

49.- BADESCH D B, ABMAN S H, AHEARN G S, BARST R J, MC CRORY D C, SIMONNEAU G, et al. Medical therapy for pulmonary arterial hypertension. ACCP evidence based clinical practice guidelines. Chest 2004; 126; 35S-62S.

50.- MICHELAKIS E D, TYMCHAK W, NOGA M, WEBSTER L, WU X, LIEN D, et al. Long-term treatment with oral sildenafil is safe and improves functional capacity and hemodynamics in patients with pulmonary arterial hypertension. Circulation 2003; 108: 2066-9.

51.- GALIE N, GHOFRANI H A, TORBICKI A, BARST RJ, RUBIN L J, BADESH D, et al. Sildenafil citrate therapy for pulmonary arterial hypertension. New Engl J Med 2005; 353: 2148-57.

52.- SITBON O, HUMBERT M, NUNES H, PARENT F, GARCIA G, HERVÉ P, et al. Long-term intravenous epoprostenol infusion in primary pulmonary hypertension: prognostic factors and survival. J Am Coll Cardiol 2002; 40: 780-8.

53.- MCLAUGHLIN V V, SHILLINGTON A, RICH S. Survival in primary pulmonary hypertension: the impact of epoprostenol therapy. Circulation 2002; 106: 1477-82.

54.- MCLAUGHLIN V V, SITBON O, BADESCH D B, BARST R J, BLACK C, GALIÈ N, et al. Survival with firstline bosentan in patients with primary pulmonary hypertension. Eur Respir J 2005; 25: 244-249.

55.- RUBIN L J, BADESCH D B, BARST R J, GALIE N, BLACK C M, KEOGH A, et al. Bosentan therapy for pulmonary arterial hypertension. N Engl J Med 2002; 346: 896-903.

Correspondencia a:

Dr. Iván Caviedes Soto

Av. Vitacura $5951,7^{\circ}$ piso.

Fono: 2101101 - Fax: 2101082

E-mail: icaviedes@alemana.cl 